\title{
Negative trial results are important to understand the mechanisms of multimodality therapy in gastric cancer
}

\author{
Christoph Schuhmacher
}

Published online: 1 July 2011

(c) The International Gastric Cancer Association and The Japanese Gastric Cancer Association 2011

Several factors influence the outcome of gastric cancer patients with invasive growth beyond the submucosa, which includes lymphatic and concomitant occult, intraperitoneal tumor cell spread. Organ metastasis or tumor dissemination outside the abdominal cavity is a late finding in gastric cancer compared to other tumor entities.

Therapy of gastric cancer thus has to address this fact not only by the complete removal of the primary lesion (R0 resection), but at the same time by the adequate treatment of the routes of tumor cell dissemination. The dissection of the gastric lymph node basins at the time of primary tumor resection constitutes the surgical, and probably most important, part of this treatment. However, invisible tumor spread outside the resectable plane, whether it be peritoneal or within the abdominal organs and tissue, needs additional consideration.

Before evaluating additional modalities to tackle this disease-inherent problem, the importance of the experience of the treating surgeon should be stressed. Surgical quality seems to be the most important factor influencing longterm patient survival and sets the benchmark against which all additional (neo) adjuvant treatments must be compared.

Historically, postoperative application of chemotherapy has been used first to address the poor outcome of gastric cancer patients (i.e., adjuvant chemotherapy if given after an $\mathrm{R} 0$ resection).

This editorial refers to the article doi:10.1007/s10120-011-0027-3.

C. Schuhmacher $(\bowtie)$

Department of Surgery,

Technische Universität München, Munich, Germany

e-mail: Schuhmacher@chir.med.tu-muenchen.de
This approach aims to eliminate any minimal residual tumor, which, at the time of initial treatment, is not detectable by diagnostic means.

After two decades of clinical trials and two meta-analyses with inconsistent results, the concept of the preoperative application of chemotherapy was introduced (neoadjuvant chemotherapy). In contrast to adjuvant treatment, neoadjuvant chemotherapy aims primarily at downsizing the primary lesion in order to increase the chance of a complete tumor resection. In the initial trial to test this hypothesis, polychemotherapy-based on cisplatinum-was used, comparable to those known from many previous adjuvant trials.

In addition to cytostatic medication, radio-chemotherapy has been evaluated, to improve the local tumor control and thus prolong survival.

Three European studies (MAGIC, ACCORD, EORTC 40954), one American trial (SWOG INT 0116), and one Japanese trial (ACTS GC) have addressed these options in the past decade, all with the highest level of methodological evidence. None of the regimens used targeted the intraperitoneal cavity, however, a clinical fact of great importance, which has now been addressed by the Japan Clinical Oncology Group trial (JCOG) 9206-2 reported in this issue of Gastric Cancer.

The neoadjuvant application of chemotherapy in all the above-mentioned trials improved the resectability of the primary lesion compared to the surgery-alone arm. Also, all the above-mentioned trials, with the exception of the EORTC 40954 study, showed a statistically significant improvement in survival in the multimodality-treatment arm.

In addition, in the United States, radiotherapy, and in Japan, adjuvant chemotherapy, did result in a better outcome if compared to surgery alone.

With this short overview in mind, how should we interpret the final results of the JCOG trial 9206-2? 
Addressing intraperitoneal dissemination is a crucial factor when planning concepts not only to improve survival but also to improve the quality of life of patients with intraabdominal recurrent disease. Although cisplatin has been delivered intraperitoneally by other groups, mainly in patients with ovarian cancer, to date there is no standard intraperitoneal cytotoxic treatment available. The ideal cytotoxic regimen should cause no local damage to the peritoneum itself, it should be rapidly metabolized to nontoxic metabolites in the liver, it should have a slow exit from the peritoneal cavity, and of course it should have well-described experimental evidence of a concentrationdependent cytotoxicity against the tumor.

The biological matrix of intraabdominal tumor cells is different compared to that of tumor cells embedded in vascularized organs or soft tissue. Thus, other mechanisms of tumor-cell-specific attachment need to be considered. Tumor-targeted monoclonal antibodies might resolve a number of the problems inherent to intraperitoneal chemotherapy, as presently under investigation using a trifunctional antibody (catumaxomab).

It is noteworthy that, as in the present trial, postoperative chemotherapy cannot be delivered in all patients, due to numerous postoperative complications, which include reduced state of health, lack of compliance, etc. The intraoperative addition of cytotoxic treatment, on the contrary, should be feasible in practically all patients. Furthermore, this approach might sufficiently destroy cancer cells that might have been set free by manipulation of the tumor or the dissection of the lymphatic drainage.

An important question in trials showing no difference between the standard and the experimental arm is how to interpret these, in the first moment, discouraging results.

An answer to this dilemma can be to compare the results with those of other trials. The British MAGIC study, the European reference trial for preoperative therapy in gastric and junctional (cardia) cancer, did show an improvement of median survival from 20 months after surgery alone to 24 months using the multimodality approach, corresponding to a 5-year survival of 23 versus $36.3 \%$.

JCOG 9206-2, in contrast, did manage to achieve an overall survival of $60.9 \%$ in the surgery alone-arm and $62 \%$ in the multimodality treatment arm. These results are in accordance with the findings of EORTC 40954, another randomized trial, which did not show a significant benefit for the multimodality treatment arm, but showed an excellent outcome for the surgery-alone control arm. On this basis one might well speculate that, after an adequate surgical intervention, additional perioperative chemotherapy, as it is presently available, no matter which route of application is chosen, will not contribute significantly to the long term-survival of gastric cancer patients.
However, the results of the Japanese ACTS-GC trial were able to demonstrate a significant benefit of an adjuvant chemotherapy concept. Even after an adequate surgical procedure with optimal results for surgery as the only treatment (control) the multimodality arm did show an improved survival. For this reason it is of importance to follow this concept and invest in the investigation of the optimal drug combination, timing, and route of application of any multimodality concept.

Furthermore, in the era of the individualization of treatment, it is of outstanding importance to get more accurate information about the stage of the disease. Here an evaluation of not only the classical TNM categories would be useful, but, more than that, information about occult intraperitoneal cell dissemination and other until now unknown parameters (e.g., circulating blood, bone marrow, distant lymphatic tissue, diaphragmatic milky spots) should become available.

In addition to this sophisticated staging, translational research has to combine efforts to predict response to cytotoxic therapy on the basis of biopsied tissue derived from the primary tumor. Given the assumption that only around $20-25 \%$ of all tumors will show a response to chemotherapy, it is our responsibility as physicians to prevent the application of ineffective and potentially toxic polychemotherapy in approximately $75-80 \%$ of gastric cancer patients.

Beyond this biological statement, localization of the tumor matters as well. In a recently presented meta-analysis (ASCO 2010) of randomized trials regarding multimodality treatment of esophageal and gastric cancer it was shown that mainly tumors in the esophagogastric junction showed benefit from neoadjuvant chemotherapy. Tumors of the stomach had only a minimal benefit. One might speculate that junctional tumors are surgically more demanding due to the narrow anatomical window in this area between the abdomen and mediastinum.

Research in this field continues. Based on the unprecedented discipline of Japanese clinical research groups it will be a question of time as researchers proceed rapidly towards the customized treatment of gastric cancer in the near future.

One message is clear. Surgeons operating on gastric cancer patients, especially outside Japan, should spare no effort to improve their technical skills towards excellence in radical and appropriate lymphadenectomy. Last, but not least, they must limit their complication rates to a minimum, even with rising numbers of multi-morbid and older patients.

Benchmarking of our own results with those of Japanese standards is reasonable, and sooner or later difference in outcome (quality of treatment) should find its place in a performance-related remuneration system. 
Given the fact that pharmaceutical progress is extremely costly and even by using innovative and promising biological approaches the results quite often end in only marginal survival benefit, excellence in surgery should be promoted, since it is available for the price of dedicated teaching. In the long run, especially in times of health budget cuts, this approach will pay off for doctors, hospitals, and patients.
Finally, it is always of great importance to critically evaluate what we are doing in healthcare. Thus, studies about interventions that we previously thought of as useful, but that turn out not to be, have informative content equal to that of studies with positive results. This idea prompted the headline: "Applause for the publishing of negative trial results" in the Financial Times issue of November 2009.

We should fully agree with this sentiment. 